\title{
N6-Methyladenosine-Sculpted Regulatory Landscape of Noncoding RNA
}

\author{
Zhongyuan Zhang ${ }^{1 \dagger}$, Wei Wei ${ }^{2 \dagger}$, Hao Wang ${ }^{2 *}$ and Jiangning Dong ${ }^{1 *}$ \\ ${ }^{1}$ Department of Radiology, The First Affiliated Hospital of the University of Science and Technology of China, Anhui Provincial \\ Cancer Hospital, Hefei, China, ${ }^{2}$ Department of Laboratory Medicine, Division of Life Sciences and Medicine, The First \\ Affiliated Hospital of the University of Science and Technology of China, Hefei, China
}

OPEN ACCESS

Edited by: Jian-ye Zhang,

Guangzhou Medical University, China

Reviewed by:

Ki-Jun Yoon,

Korea Advanced Institute of Science and Technology, South Korea

Dong Ren,

The First Affiliated Hospital of Sun

Yat-sen University, China

*Correspondence: Hao Wang

wanghao986118@163.com Jiangning Dong dongjn@163.com

${ }^{\text {t}}$ These authors have contributed equally to this work

Specialty section: This article was submitted to Molecular and Cellular Oncology, a section of the journal

Frontiers in Oncology

Received: 19 July 2021 Accepted: 27 September 2021 Published: 15 October 2021

Citation:

Zhang Z, Wei W, Wang $\mathrm{H}$ and Dong $J$

(2021) N6-MethyladenosineSculpted Regulatory Landscape of Noncoding RNA.

Front. Oncol. 11:743990. doi: 10.3389/fonc.2021.743990
The exploration of dynamic N6-methyladenosine (m6A) RNA modification in mammalian cells has attracted great interest in recent years. M6A modification plays pivotal roles in multiple biological and pathological processes, including cellular reprogramming, fertility, senescence, and tumorigenesis. In comparison with growing research unraveling the effects of m6A modifications on eukaryotic messenger RNAs, reports of the association between noncoding RNAs and m6A modification are relatively limited. Noncoding RNAs that undergo m6A modification are capable of regulating gene expression and also play an important role in epigenetic regulation. Moreover, the homeostasis of m6A modification can be affected by noncoding RNAs across a broad spectrum of biological activities. Importantly, fine-tuning and interaction between these processes are responsible for cell development, as well as the initiation and progression of the disease. Hence, in this review, we provide an account of recent developments, revealing biological interactions between noncoding RNAs and m6A modification, and discuss the potential clinical applications of interfering with $\mathrm{m} 6 \mathrm{~A}$ modification.

Keywords: $\mathbf{N}^{6}$-methyladenosine (m6A), post-transcriptional modification, noncoding RNAs, cancer, regulation

Abbreviations: AGO2, Argonaut RISC catalytic component 2; ALKBH5, Alkylated DNA repair protein AlkB homolog 5; CDCP1, CUB domain containing protein 1; DGCR8, Di George syndrome critical region 8; eIF3, Eukaryotic translation initiation factor 3; EMT, Epithelial-mesenchymal transition; FAM225A, Family with sequence similarity 225 member A; FMR1, Fragile X mental retardation protein translational regulator 1; FOXM1, Forkhead box M1; FTO, Fat mass and obesity associated protein; GAS5, Growth arrest special 5; HAKAI, Also known as CBLL1, Cbl protooncogene like 1; HBXIP, Hepatitis BX-interacting protein; HDGF, Hepatoma-derived growth factor; HNRNP, Heterogeneous nuclear ribonucleoprotein; HNRNPA2/B1, Heterogeneous nuclear ribonucleoprotein A2/B1; IGF2BP1/2/3, Insulin-like growth factor 2 mRNAbinding protein 1/2/3; IRES, Internal ribosome entry site; ITGB3, Integrin subunit beta 3; KCNK15-AS1:KCNK15 and WISP2 antisense RNA 1; MALAT1, Metastasis-associated lung adenocarcinoma transcript 1, also known as NEAT2; MAPK, Mitogen activated kinase-like protein; MEG3, Maternally expressed 3; mESC, Mouse embryonic stem cells; METTL14, Methyltransferase like 14; METTL3, Methyltransferase like 3, also known as MT-A70; NEAT1, Nuclear paraspeckle assembly transcript 1; NKAP, NFKB activating protein; ORF, Open reading Frame; PCAT6, Prostate cancer associated transcript 6; PVT1, Plasmacytoma variant translocation 1; RBM15, RNA binding motif protein 15; VIRMA, Vir-like m6A methyltransferase associated, also known as KIAA1429; WTAP, Wilms tumor 1-associating protein; XIST, X-inactive specific transcript; YAP, Yes-associated protein; YTHDC1, YTH domain containing 1; YTHDF1, YTH N6methyladenosine RNA binding protein 1; ZC3H13, Zinc finger CCCH-type containing 13; TRIM25, tripartite motif containing 25; FAAH, fatty acid amide hydrolase. 


\section{INTRODUCTION}

Since the definition of the term "epitranscriptomics", more than 100 types of RNA modifications have been recognized in living organisms. As an important mechanism of epitranscriptomics, first characterized in the 1970s, N6-methyladenosine (m6A) is the most frequently observed internal chemical modification in eukaryotic mRNA (1-3). As illustrated by a large body of research, m6A RNA modification modulates sophisticated RNA processes, including splicing, nucleic transport, degradation, and translation efficiency, thereby broadening the diversity of RNA modification (1-3). Preferentially found in the brain, heart, and kidney, and highly conserved between humans and mice, m6A modification mainly lies in the 3'-UTR of mRNAs, near the stop codons and within internal exons (1). m6A is a dynamic and reversible event, that is manipulated selectively by enzymes that play the part of "writers", "erasers", and "readers". These effectors are equipped with multifaceted and tunable properties based on the cellular context. For certain RNA, the accessibility and biological activity of m6A modification very likely influences the outcome of physiological or pathological processes.

\subsection{Biological Role of m6A Modification 1.1.1 Writers}

m6A modification is regulated by a methyltransferase complex, the core subunits of which are composed of METTL3 and METTL14, as well as other auxiliary cofactors, including WTAP, VIRMA, RBM15/ 15B, ZC3H13, and HAKAI. METTL3, the first identified methyltransferase, often forms a heterodimer with METTL14, which functions as a conformational switch for the catalytic activity of METTL3 (2). The METTL3-METTL14 complex accurately binds to targets with the assistance of WTAP, a regulatory subunit without catalytic a domain. Interestingly, METTL3 can stimulate the translation of a set of oncogenes independently of m6A modification in lung cancer (3). VIRMA acts as an RNA-binding protein related to splicing and processing (3). The interaction of RBM15 and its paralog RBM15B with METTL3 is dependent on WTAP, both RBM15 and RBM15B bind to the U-rich sequence near the m6A sites (4). Correct nuclear localization of the writer complex relies on $\mathrm{ZC} 3 \mathrm{H} 13$, which modulates the differentiation of mouse embryonic stem cells and nuclear RNA m6A methylation (5). HAKAI, an E3-ligase for E-cadherin, shares several targets with WTAP and is closely related to epithelial-mesenchymal transition (EMT) (6). Another effector of m6A modification, METTL16, other than being the $\mathrm{m} 6 \mathrm{~A}$ reader of $\mathrm{U} 6 \mathrm{snRNA}$, also mediates atypical $\mathrm{m} 6 \mathrm{~A}$ modifications. Most of the m6A residues are found in introns (7), suggesting that METTL16 probably binds to pre-mRNAs in addition to small nuclear RNA (Figure 1).

\subsubsection{Erasers}

m6A modification is reversible due to the activity of two erasers, FTO and ALKBH5, which remove m6A modification from modified RNAs. While the former is predominantly expressed in adipose tissue and hypothalamic nuclei (8) and are related to human obesity and energy metabolism (6), the latter is mainly distributed in the testis, and its depletion leads to aberrant spermatogenesis (9). Given their different localization, FTO and ALKBH5 may function separately in a tissue-specific manner (3) (Figure 1).

\subsubsection{Readers}

The fate of m6A-located transcripts is determined by a series of readers. The YTH (YT521-B homology) family was the first identified class of readers recognizing sites of m6A modifications.

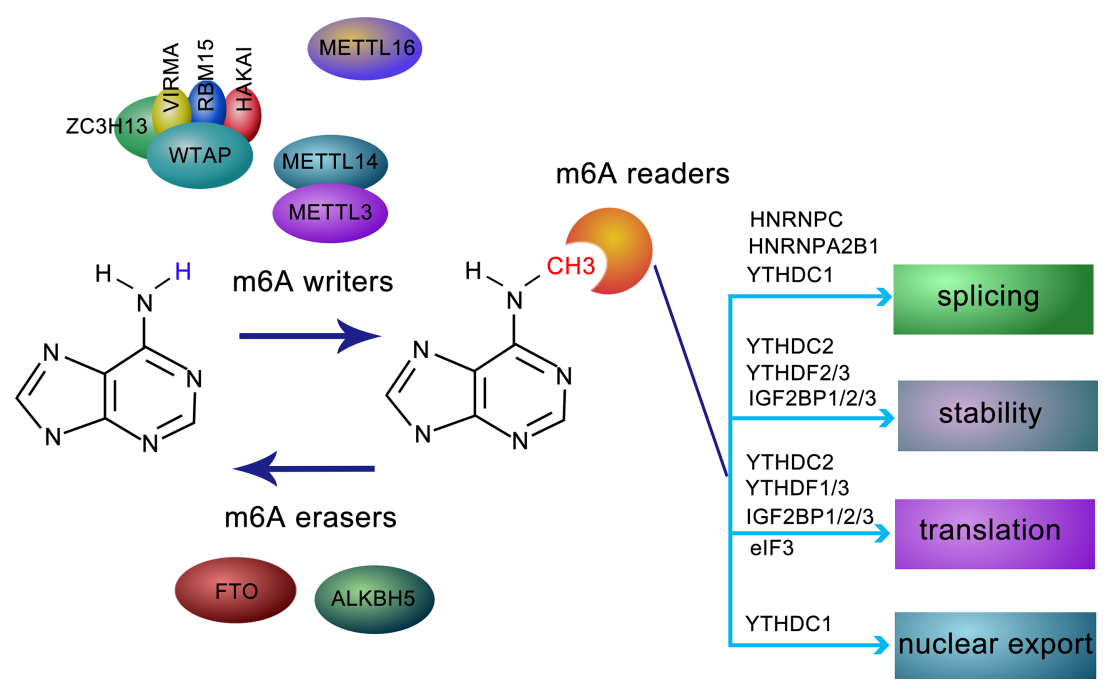

FIGURE 1 | Molecular composition and regulation mechanism of m6A methylation modification. m6A methylation is a dynamic and reversible process coordinated by methyltransferases (defined as "writers", including METTL3, METTL14, WTAP, ZC3H13, HAKAI, VIRMA, and RBM15), demethylases (defined as "erasers", FTO and ALKBH5), and "readers", such as YTHDF1-3, YTHDC1, IGF2BPs, HNRNPC, HNRNPA2B1, and elF3, recognize and bind to m6A-modified RN and mediating RNA splicing, stability, translation, and RNA nuclear export. 
Among these, YTHDC1 regulates splicing by recruiting splicing factors (4). While YTHDF1 and YTHDC2 elevate the translation efficiency of m6A-modified RNA, YTHDF2 and YTHDF3 prompt the decay of targeted RNAs (10). In mammalian germ cells, YTHDC2 is essential for meiosis depending on m6A modifications (11). Insulin-like growth factor 2 mRNA-binding proteins $1 / 2 / 3$ (IGF2BP1/2/3), a family of newly discovered m6A readers, modulate $\mathrm{m} 6 \mathrm{~A}$-transcripts localization, stability, and translational efficiency (12). Different from the abovementioned counterparts, FMR1 is an indirect and sequence-contextdependent m6A reader that inhibits translation (13). HNRNPA2B1, an HNRNP family protein, accelerates miRNA processing after recognizing m6A-pri-miRNA and attracting accessory microprocessors and triggers alternative splicing similar to the activity of METTLs on mRNA (14). HNRNPC is involved in the alternative splicing of m6A-transcripts indirectly. Eukaryotic initiation factor 3 (eIF3) contributes to ribosome loading by binding to m6A-modified sequences (15). Recently, it has been reported that NF-kappaB activating protein (NKAP) preferentially binds to m6A-pri-miR-25 rather than pri-miR-25 (16), indicating a role for the $\mathrm{m} 6 \mathrm{~A}$ reader NKAP in pancreatic cancer (Figure 1).

\subsection{Biological Role of Noncoding RNA}

The majority of human transcription products are non-coding RNAs (ncRNA) that are ubiquitously expressed in a broad spectrum of tissues (17). These RNAs engage in intricate gene expression processes, such as RNA splicing and protein translation, although they have little or no capacity for proteincoding. Recently, by virtue of the advancement in detection methods, numerous noncoding transcripts that were previously overlooked and merely regarded as intermediaries of protein synthesis, have been characterized to be critical for the posttranscriptional regulation of transcriptome expression (17). In general, ncRNAs mainly contain long noncoding RNA (lncRNA), microRNA (miRNA), circular RNA, rRNA, tRNA, and snRNA. Considering the vital role of the first three ncRNA listed above, this review describes their functions in multiple biological and pathological processes.

\subsubsection{Long Noncoding RNA}

LncRNA, consisting of over 200 nucleotides, can be divided into five types and defined as intergenic lncRNAs, intronic lncRNAs, antisense lncRNAs, bidirectional lncRNAs, and enhancer lncRNAs, according to their genomic organization (17). LncRNAs, whose exact source remains obscure at present, execute the molecular functions as decoys, guides, scaffolds, and signals (18). Widely expressed in eukaryotes, they were previously defined as anomalies in the process of transcription. However, it has now been universally accepted that lncRNAs modulate various processes of gene transcription or posttranscription via interacting with mRNAs, miRNAs, or proteins (19). Approximately half of IncRNAs are believed to be retained in the nucleus and fine-tune the chromatin spatial architecture (19), or interact with the chromosome related proteins constituting the RNA-DNA complex. Moreover, recent studies have shown that some cytoplasmic lncRNAs migrate and act in ribosomes (20). Importantly, lncRNAs are very likely to act as competing endogenous RNA (ceRNA) in regulating the repression effects of miRNAs by competitively binding to miRNAs (21).

\subsubsection{MicroRNA}

As a class of endogenous non-coding single-stranded RNA comprising 21-24 nucleotides, miRNAs function as sequencespecific negative regulators in post-transcriptional gene silencing by recognizing target $\mathrm{mRNAs}$ and mediating mRNA cleavage or translational repression (22). miRNAs are produced from primary miRNAs (pri-miRNAs), which differ in length and are located in the introns of host genes. Pri-miRNAs are first converted into hairpin-shaped precursor microRNA (pre-miRNA) upon the cleavage of Drosha (23). Afterward, pre-miRNAs translocate from the nucleus to the cytoplasm where Dicer digests premiRNA to generate functionally mature miRNA (24). The nucleotides $2-8$ at the 5 '-end of miRNAs decide the binding sequence of $\mathrm{mRNA}$, hundreds of mRNAs can be targeted by a single miRNA, and vice versa, each mRNA can also be subjected to regulation by different miRNAs simultaneously or sequentially, this phenomenon may be explained by the imperfect base-pairing between miRNAs and mRNAs, and because most binding sites are positioned within the 3'-UTR of mRNAs (25).

\subsubsection{Circular RNA}

Circular RNA (circRNA), characterized by the lack of free 5' and 3 ' ends, is a novel class of ncRNAs generated from non-canonical back-splicing or the exon skipping of linear pre-mRNAs (26). CircRNAs are mostly composed of internal exons and reside predominantly in cytoplasm. Intriguingly, compared to their linear counterparts, the covalently closed loop structure endows circRNAs with preferable stability (27).

The degradation of circRNAs is solely dependent on endoribonucleolytic cleavage owing to the absence of the 5' cap and 3' poly(A) tail (18). The resistance to RNA exonuclease or RNase $\mathrm{R}$ hinders their degradation and enhances their detectability. Another property of circRNAs lies in their specific expression in certain cell types and tissues. In addition, circRNAs are sensitive to cellular stress. All these features render the potential of circRNAs to be novel molecular biomarkers for the diagnosis of diseases (4).

\section{LncRNA AND N6-METHYLADENOSINE}

Similar to mRNAs, lncRNAs are subjected to m6A methylation in various cell lines. $\mathrm{m} 6 \mathrm{~A}$ residues show a preference for locating in lncRNA transcripts that have been subjected to alternative splicing (19), indicating that m6A deposition may play a potential role in the formation of IncRNA isoforms. m6A methylation sites are welldistributed along transcripts, in comparison to mRNAs modification patterns (27). As illustrated by recent studies, the abundance of m6A-modified lncRNAs dramatically decreases in human fetal tissues (21), in comparison with mRNA (Figure 2 and Table 1). 
A

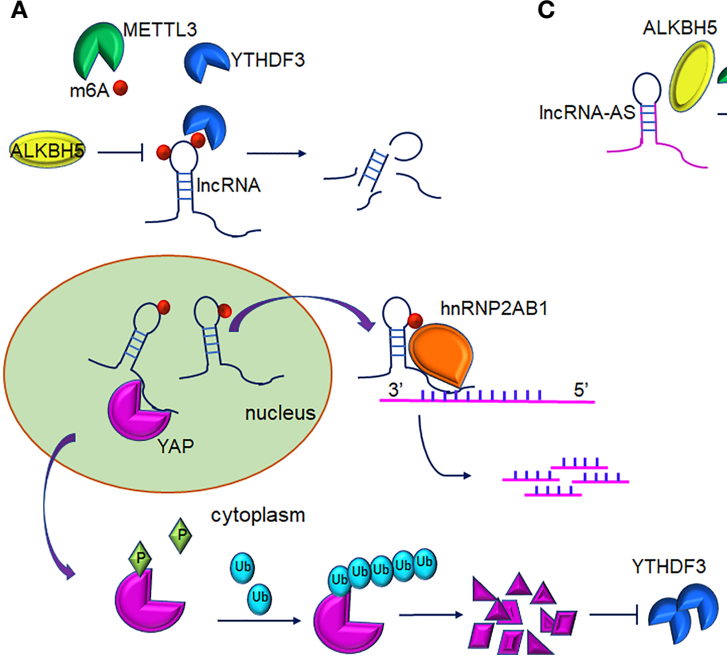

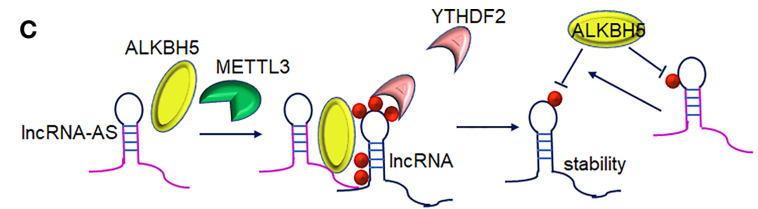

B

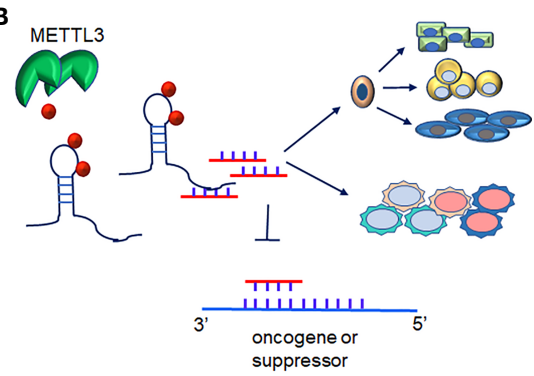

FIGURE 2 | Interplay between IncRNAs and m6A modification. (A) m6A-mediated IncRNAs target mRNA/protein. m6A-IncRNA GAS5 targets endogenous YAP, which induces YAP degradation and downregulation of its target YTHDF3. In turn, YTHDF3 promotes degradation of m6A-modified GAS5. m6A-IncRNA RP11 binds to the mRNA of FBXO45 and SIAH1 promoting their degradation. (B) m6A-mediated methylation of IncRNA-miRNAs activity in gene expression. METTL3-mediated m6A-IncRNAs methylation serves as a sponge to restrain miRNA activity and thus abolishes miRNAs-modulated mRNA inhibition. Furthermore, interaction of IncRNA and miRNA contribute to proper differentiation of mouse embryonic stem cells and the inflammatory response of cells. (C) The role of LncRNA-AS in m6A-mediated regulation. LncRNA can directly interact with ALKBH5 or METTL3. GAS5-AS binds to m6A-GAS in a YTHDF2-dependent manner and reduces its stability. Furthermore, demethylation of GAS5 is mediated by ALKBH5 and GAS-AS facilitate this process. ARHGAP5-AS1 can recruit METTL3 to stimulate the m6A modification of ARHGAP5 and increase its stabilization in the cytoplasm.

\section{1 m6A-Mediated Regulation on LncRNA Expression}

The dysregulation of lncRNAs has been proven to play a nonnegligible role in tumorigenesis. In colorectal cancer (CRC) cells, increased expression of IncRNA RP11 in the nucleus and chromatin is mediated by m6A methylation. RP11 is capable of directly binding to the mRNA of Siah1 and Fbxo45 and then stimulates mRNA degradation, leading to the post-translational stabilization of ZEB1 (19). As revealed by Ni et al., m6A methylation participates in the regulation of YAP signaling in the progression of CRC. IncRNA GAS5 inhibits CRC progression via mediating the phosphorylation and degradation of YAP. However, the interaction of GAS5 and the m6A reader YTHDF3 results in the degradation of the former, contributing to the suppression of cell proliferation and metastasis abilities (28) (Figure 2A). It is of note that lncRNA can also interact with m6A writers such as METTL3. In gastric cancer, LINC00470 associates with METTL3 to weaken the stabilization of PTEN mRNA. YTHDF2 accounts for the detection of m6A sites within PTEN mRNA (38). In addition, lncRNA NEAT1 is demethylated by ALKBH5, consequently upregulates and prompts the invasion and metastasis of GC cells (29). In addition, In osteosarcoma, m6A demethylation on lncRNA PVT1 is mediated by ALKBH5 and associated with malignant properties (30).

Patil et al. demonstrated that the knockdown of METTL3 impaired the silencing of gene transcription in human cells. This process was modulated by lncRNA XIST (4). An enhanced abundance of m6A modification on XIST residues is mediated by RBM15/15B, which recruits the WTAP-METTL3 complex, and subsequently, YTHDC1 preferentially binds to $\mathrm{m} 6 \mathrm{~A}$ residues on XIST. Methyltransferase METTL16 can interact with lncRNA MALAT1, a cancer-related lncRNA $(7,48,49)$. The crosslinking sites mainly occur at the 3' UTR of MALAT1, wherein the triple helix element is usually recruited. m6A methylation disrupts the local RNA architecture of MALAT1 (50) and impedes the binding of RBPs to MALAT1. The aberrant expression of IGF2BP2 has been associated with insulin resistance, diabetes, and even neoplasia (51). IGF2BP2 interacted with lncRNA DANCR modified by $\mathrm{m} 6 \mathrm{~A}$, resulting in the stability enhancement of DANCR. IGF2BP2 and DANCR jointly contribute to the proliferation and stemness-like properties of pancreatic cancer cells (31).

\section{2 m6A-Mediated LncRNA-miRNA Interaction}

Based on genome-wide microarray analysis, a novel upregulated oncogenic lncRNA FAM225A was found to be significantly related to recurrence and distant metastasis in nasopharyngeal carcinogenesis (NPC) (32). Enhanced stabilization of FAM225A likely resulted from the enrichment of $\mathrm{m} 6 \mathrm{~A}$ modifications within its transcripts. Most FAM225A is located in the cytoplasm and absorbs miR-590-3p and miR-1275, resulting in the upregulation of their common target integrin $\beta 3$ (ITGB3). ITGB3 was shown to account for malignant phenotype progression in NPC cells (32). Yang et al. found that lncRNA 1281 could sequester pluripotency-related let-7 family miRNAs in mouse embryonic 
TABLE 1 | LncRNAs in disease or normal cells and related m6A effectors.

\begin{tabular}{|c|c|c|c|c|c|}
\hline LncRNA & Cancer & Expression & Pathway & m6A effector & Reference \\
\hline GAS5 & $\mathrm{CRC}$ & Downregulation & YAP & YTHDF3 & (28) \\
\hline NEAT1 & GC & Upregulation & EZH2 & ALKBH5 & (29) \\
\hline PVT1 & OS & Upregulation & - & $\begin{array}{l}\text { ALKBH5 } \\
\text { YTHDF2 }\end{array}$ & (30) \\
\hline MALAT1 & mESC & - & - & METTL16 & $(7)$ \\
\hline DANCER & PC & - & - & IGF2BP2 & (31) \\
\hline FAM225A & NPC & Upregulation & ITGB3/FAK/PI3K/AKT & METTL3 & (32) \\
\hline Linc1281 & mESC & - & Lin28 & METTL3 & (33) \\
\hline Linc00958 & $\mathrm{HCC}$ & Upregulation & SREBP1/FASN/SCD1/ACC1/HDGF & METTL3 & (34) \\
\hline BLACAT2 & GC & Upregulation & - & METTL3 & (39) \\
\hline Olfr29-ps1 & MDSCs & Upregulation & IL-6/MyD88 & METTL3 & (40) \\
\hline LINC00958 & $\mathrm{BC}$ & Upregulation & YY1 & METTL3 & (41) \\
\hline GAS5-AS1 & $\mathrm{CC}$ & Downregulation & - & $\begin{array}{l}\text { YTHDF2, } \\
\text { ALKBH5 }\end{array}$ & (42) \\
\hline FOXM1-AS & GBM & Upregulation & sox2 & ALKBH5 & (43) \\
\hline KCNK15-AS1 & $\mathrm{PC}$ & Downregulation & - & ALKBH5 & (44) \\
\hline ABHD11-AS1 & NSCLC & Upregulation & - & METTL3 & (45) \\
\hline ARHGAP5-AS1 & $\mathrm{GC}$ & Upregulation & SQSTM1 & METTL3 & (46) \\
\hline HOTAIR & NSCLC & - & - & m6A signal & (47) \\
\hline
\end{tabular}

CRC, colorectal cancer; GC, gastric cancer; OS, osteosarcoma; mESC, mouse embryonic stem cells; PC, pancreatic cancer; NPC, nasopharyngeal carcinoma; HCC, hepatocellular

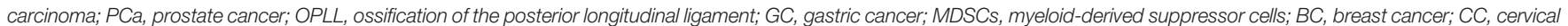
cancer; GBM, glioblastoma; PC, pancreatic cancer; NSCLC, non-small cell lung cancer.

stem cells (mESC) (33). Sufficient m6A modification on lncRNA 1281 transcripts was necessary for this direct RNA-RNA interaction, which maintained mESC markers and proper differentiation (Figure 2B and Table 1). Studies have revealed that lncRNA 00958 acts as an oncogenic gene in gliomagenesis (52), whereas its role in hepatocellular carcinoma (HCC) was not revealed until very recently. Zuo et al. disclosed that METTL3 induced the upregulation of lncRNA 00958, which exerted lipogenesis and the unfavorable survival of HCC patients (34). LncRNA 00958 could sponge miR-3619-5p whose target was hepatoma-derived growth factor (HDGF). The inhibitory effects of miR-3619-5p in the dissemination and invasion of HCC cells have also been described previously (53). In addition, m6 A modification is also involved in nontumor pathologic or physiological processes, including heart and brain ischemia reperfusion injury (54). LncRNA MALAT1 targets miR-26b in the regulation of PTGS2, and has been reported to exacerbate inflammatory response in myocardial ischemia infarction patients, while m6A modification may also enhance MALAT1 expression (55). The IncRNA-BLACAT2 is a sponge of miR$193 \mathrm{~b}-5 \mathrm{p}$ and has been associated with the progression of gastric cancer while silencing BLACAT2 inhibited cancer cells migration and invasion. A further study showed that METTL3 was a direct target of miR-193b-5p (39). Furthermore, similar to ceRNA, the lncRNA pseudogene Olfr29-ps1 sponged miR-214-
$3 \mathrm{p}$ and influenced IL6-mediated m6A modification, which jointly modulated the differentiation of myeloid-derived suppressor cells (40). Likewise, METTL3-mediated LINC00958 upregulation also played a similar ceRNA role over miR-378a-3p to promote YY1 expression in BC tumorigenesis (41). METTL3 induced LncRNA MEG3 stability and suppressed the progression of HCC by targeting miR-544b/BTG2 signaling (35). Furthermore, m6A methylation on lncRNA PCAT6 contributed to PCAT6 upregulation in an IGF2BP2-dependent manner in prostate cancer (36). Yuan et al. also showed that METTL3 promoted osteogenic ossification through the upregulation of IncRNA XIST, and further investigation confirmed that IncRNA XIST regulated osteogenic differentiation of primary ligament fibroblasts via miR-302a3 , which targets ubiquitin-specific protease 8 (USP8) (37).

\section{3 m6A-Methylation of LncRNA-Antisense ncRNA}

A recent study showed that GAS5-AS1, the antisense RNA of GAS5, was downregulated in HCC (56) and non-small cell lung carcinoma (NSCLC) (57). In cervical cancer (CC) cells, the lncRNA GAS5-AS1 attenuates m6A modification of GAS5 through antisense pairing with the GAS5 3' UTR, and thus, epigenetically enhanced GAS5 stability in a YTHDF2-dependent fashion. Whereas knockdown GAS5-AS1 resulted in larger 
tumors, enhanced metastasis, and advanced prognosis in CC patients (42). The lncRNA GAS5-AS1 also interacts with ALKBH5 to induce GAS5 upregulation (Figure 2C and Table 1). Analogously, the IncRNA ARHGAP5-AS1 can recruit METTL3 to methylate ARHGAP5 mRNA in gastric cancer cells, thus, increased levels of m6A-ARHGAP5 were predictive of enhanced chemoresistance and poor prognosis (46). Zhang et al. revealed that FOXM1 maintained self-renewal and tumorigenic properties of glioblastoma stem-like cells (GSCs) (43). ALKBH5-mediated demethylation of nascent FOXM1 transcripts and upregulation of FOXM1 expression was facilitated by lncRNA FOXM1-AS. GSCs properties could be destroyed significantly when ALKBH5 or FOXM1-AS expression was blocked. He et al. determined that lncRNA KCNK15-AS1 was significantly decreased in cancer cells compared to normal pancreatic ductal epithelial cells (44) and its expression was correlated with the degree of m6A methylation. Simultaneously, ALKBH5 could demethylate KCNK15-AS1 and enhance the repression effects of KCNK15-AS1 on cancer cell viability. Furthermore, METTL3 was found to be responsible for the m6A methylation on the ABHD11-AS1 transcript and enhanced its stability in NSCLC tumorigenesis (45).

Recently, the IncRNA HOTAIR was reported to act as a plasma-derived biomarker of NSCLC, and m6A methylation was found to be co-expressed with HOTAIR (47). Moreover, many other lncRNAs, such as ANRIL, NEAT1, PVT1, TUG1, and DICER1-AS1, probably undergo m6A modification (58). These findings were conducive to improving understanding of the underlying mechanisms of human diseases and designing applicable therapeutic strategies.

\section{MICRORNA AND N6- METHYLADENOSINE}

\section{1 m6A-Mediated Processing of Primary-miRNAs}

Many studies have reported that miRNAs are abnormally expressed in different pathological processes, including diabetes, neurodegenerative diseases, and carcinomas. Pri-miRNA transcript can be methylated by METTL3 in the nucleus, and contribute to the recognition of pri-miRNAs by the microprocessor protein DGCR8 (59). Consistently, METTL3 deficiency can markedly decrease the expression of mature miRNAs. In bladder cancer, for example, upregulation of METTL3 accelerated the processing of pri-miR221/222, which promoted the proliferation, migration, and invasion of cancer cells in an m6A-dependent manner (60). Mature miR221/222 was also confirmed to have a carcinogenic role in other carcinomas (61), such as prostate cancer (62) and thyroid cancer (63). Analogously to its impact on bladder carcinoma, METTL3 facilitated the pri-miR-1246 maturation process and paved the way for the enhanced metastasis of CRC cells via the MAPK signaling pathway (64). Analogously, METTL3 upregulated miR-1246 expression and contributed to NSCLC cell growth (65). Likewise, miR-25-3p maturation was impeded by m6A modification mediated by METTL3 in pancreatic ductal adenocarcinoma (16).
Furthermore, METTL3 drove the development of obstructive renal fibrosis by promoting miR-21-5p maturation (66). In vitro, the knockdown of METTL3 reduced the expression of miR-221-3p via m6A methylation of pri-miR-221-3p mRNA (67). It is of note that cigarette smoking has been shown to stimulate the METTL3 promoter resulting in its enhanced energetic transcription. Wang et al. provided evidence that METTL3-mediated miR-143-3p upregulation enhanced VASH1 repression, which thereafter triggered brain metastasis and the angiogenesis of lung cancer (68). Consistently, miR-873-5p maturation induced by exogenous METTL3 activity could protect mouse renal tubular epithelial cells (mRTECs) against colistin-induced nephrotoxicity (69). Interestingly, Yang et al. suggested that miR24-2 not only indirectly facilitated METTL3 transcription, but also enhanced miR6079 expression by promoting m6A methylation on primiR6079 in liver cancer cells (70).

Downregulation of the methyltransferase METTL14 was shown to be responsible for the aberrant m6A modification observed in HCC metastasis. miR126 is a downstream target of METTL14 and was markedly decreased in cancer tissues compared to the adjacent tissue (71). Consistently, Chen et al. revealed that downregulation METTL14 could induce primiRNA-375 processing arrest and reduced overall levels of miRNA-375 in CRC (72). Thus, miRNA-375 was revealed as an anti-oncogene regulated by METTL14 through the YAP/SP1 pathway and m6A methylation. miR-200a is also subject to METTL14 modulation (Figure 3A and Table 2). As a reader of the m6A label in pri-miRNAs, HNRNPA2/B1 widely directed the maturation of pri-miRNA in LCC9 breast cancer cells (83). This processing was $\mathrm{m} 6 \mathrm{~A}$ dependent and promoted endocrine resistance in LCC9 cells, resulting in the poor survival of patients with advanced tumors.

\section{2 miRNAs Directly Target m6A Effectors}

The transcriptional regulator SRF is regulated epigenetically and responsible for cells contractility and proliferation (84). A recent study indicated that IGF2BP1 promotes the expression of SRF in a conserved and m6A-dependent manner, IGF2BP1 can impair the miRNA-directed decay of the SRF mRNA, resulting in enhanced SRF-dependent transcriptional activity (85). A sequence pairing mechanism contributes to miRNAs regulation of m6A methylation of mRNAs. Yang et al. reported that miRNA-145 expression might be considered a negative prognostic marker in liver cancer. The $3^{\prime}$ UTR region of YTHDF2 mRNA contains direct binding sites for miR-145, and the overexpression of mir-145 increased m6A deposition and downregulated YTHDF2 expression (Figure 3B and Table 2) (73).

In hemorrhoid vascular endothelial cells, a decrease in miR4729 expression was confirmed to be responsible for vascular cell proliferation. miR-4729 was responsible for silencing METTL14 expression, reducing TIE1 mRNA stability, and inhibiting angiogenesis (74). Additionally, miR-103-3p directly targets METTL14 to inhibit osteoblast activity (75). METTL3 has also been reported to be a target of miR-186 in hepatoblastoma (76). Overexpression of miR-186 drastically ameliorated the metastatic phenotype induced by METTL3 upregulation. Moreover, as demonstrated in bladder cancer and CRC, the 


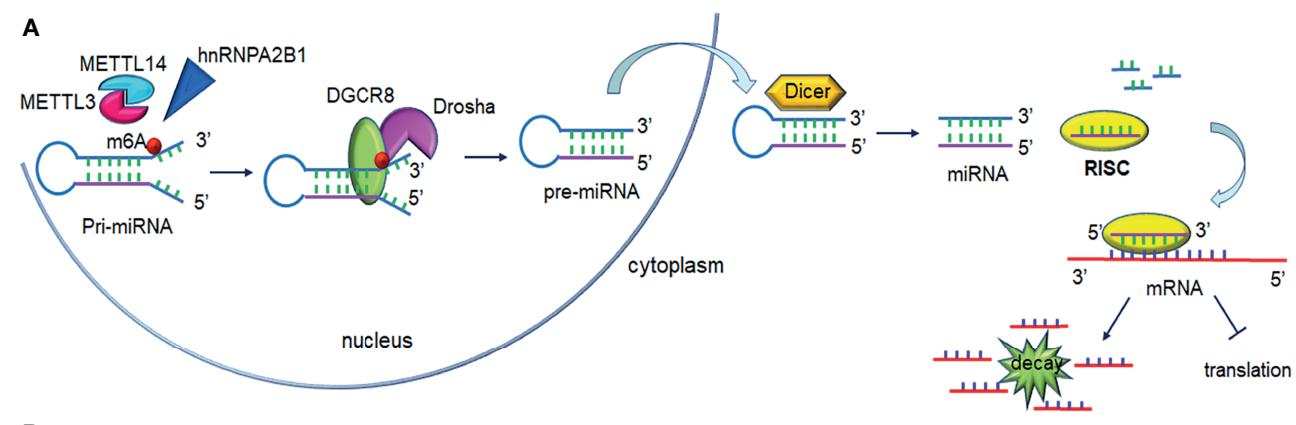

B

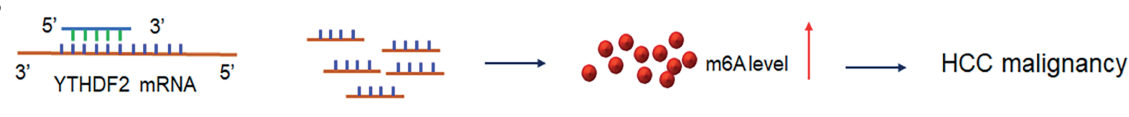

C

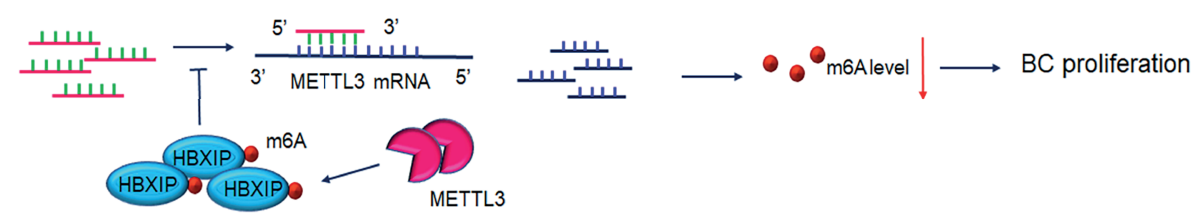

FIGURE 3 | Interplay between microRNA and m6A modification. (A) m6A modification promotes pri-miRNAs processing. MEITL3/ETTL14 facilitates DGCR8 recognition of pri-miRNAs contributing to the increased levels of mature miRNAs under the cleavage of Drosha and Dicer. One strand of the miRNAs is loaded on RISC and mediates mRNA silencing or translation repression. (B) MiRNAs regulate m6A levels through binding to m6A readers. MiRNAs bind to YTHDF2 mRNA inducing its degradation and interfere with HCC cell malignancy. (C) MiRNAs regulate m6A levels by binding to m6A writers. miRNAs degrade MEITL3 mRNA and lead to decreased breast cancer cell proliferation. Upregulation of METTL3 contributes to HBXIP m6A modification, in turn, HBXIP reverses the miRNAs-induced degradation of METTL3, forming the positive feedback of METTL3/HBXIP/miRNA/METTL3. DGCR8, DiGeorge syndrome critical region 8; RISC, RNA-induced silencing complex.

TABLE 2 | MicroRNAs in disease and related m6A effectors.

\begin{tabular}{|c|c|c|c|c|c|}
\hline MiRNA & Disease & Expression & Pathway & m6A Effector & Reference \\
\hline miR221/222 & BCA & Upregulation & PETN & METTL3 & (60) \\
\hline miR-1246 & $\mathrm{CRC}$ & Upregulation & SPRED2, Raf/MEK/ERK & METTL3 & (64) \\
\hline miR-1246 & NSCLC & Upregulation & PEG3 & METTL3 & (65) \\
\hline miR-221-3p & $\mathrm{BC}$ & Upregulation & HIPK2/Che-1 & METTL3 & $(67)$ \\
\hline miR-143-3p & NSCLC & Upregulation & VASH1NEGFA & METTL3 & (68) \\
\hline$m i R-21-5 p$ & ORF & Upregulation & SPRY1/ERK/NF-kB & METTL3 & $(66)$ \\
\hline miR-873-5p & mRTECs & - & Keap1/Nrf2 & METTL3 & (69) \\
\hline miR126 & $\mathrm{HCC}$ & Downregulation & - & METTL14 & $(71)$ \\
\hline miRNA-375 & $\mathrm{CRC}$ & Downregulation & YAP/SP1 & METTL14 & (72) \\
\hline miR-25-3p & $\mathrm{PC}$ & Upregulation & PHLPP2/AKT-p70S6K & $\begin{array}{l}\text { METTL3 } \\
\text { NKAP }\end{array}$ & (16) \\
\hline miR-145 & $\mathrm{HCC}$ & Downregulation & - & YTHDF2 & (73) \\
\hline miR-4729 & Hemorrhoids & Downregulation & TIE1NEGFA & METTL14 & (74) \\
\hline miR-103-3p & Osteoporosis & Upregulation & - & METTL14 & (75) \\
\hline miR-186 & $\mathrm{HB}$ & Downregulation & Wnt/ $\beta$-catenin & METTL3, YTHDF2 & (76) \\
\hline miR-338-5p & GC & Downregulation & EED/CDCP1 & METTL3 & $(77)$ \\
\hline miR-4443 & NSCLC & Upregulation & FSP1 & METTL3 & (78) \\
\hline $\begin{array}{l}\text { miR-24-2/ } \\
\text { miR6079 }\end{array}$ & $\mathrm{HCC}$ & Upregulation & Pim1/JMJD2A & METTL3 & (70) \\
\hline miR-33a & NSCLC & Downregulation & EGFR & METTL3 & (79) \\
\hline miR-600 & NSCLC & Downregulation & $\begin{array}{l}\text { EGFR/PI3K/AKT } \\
\beta \text {-catenin }\end{array}$ & METTL3 & (80) \\
\hline miR-29a & GBM & Downregulation & QKI-6/EGFR ERK/PI3K/AKT & WTAP & (81) \\
\hline miR-let-7g & $\mathrm{BC}$ & Downregulation & HBXIP & METTL3 & (82) \\
\hline
\end{tabular}

BCA, bladder cancer; mRTECs, mouse renal tubular epithelial cells; HB, hepatoblastoma; $B C$, breast cancer. ORF, obstructive renal fibrosis. 
oncogenic role of METTL3 substantially relied on the degradation of tumor suppressor mRNAs targeted by miRNAs. $\mathrm{Du}$ et al. confirmed that in NSCLC cells, miR-33a bound to the 3'-UTR of METTL3 mRNA, and thus, resulted in a decrease in m6A deposition and proliferation of cancer cells accompanied by the downregulation of METTL3 (79). Moreover, in gastric cancer, miR-338-5p has also been reported to target METTL3 and repress the m6A-mediated translation of CDCP1 (77). Furthermore, Wei et al. indicated that miR-600 repressed METTL3 expression and eliminated the oncogenic activity induced by METTL3 on NSCLC progression (80). MiR-4443 directly targeted METTL3 and regulated the expression of FSP1 via m6A methylation (78). It has also been reported that WTAP downregulation in GSCs was caused by miR-29a upregulation, which could interfere with the malignant potential of GSCs (81). Cai et al. demonstrated that in breast cancer cells, HBXIP increased METTL3 expression by interfering with let-7g, a miRNA binding to the 3'UTR of METTL3, which subsequently interfered with METTL3 expression (82). Interestingly, METTL3 also upregulated HBXIP expression following the marked increase of overall m6A methylation, favoring the positive feedback loop HBXIP/let-7g/METTL3/HBXIP, which strongly promoted tumor cell growth and metastasis (Figure 3C and Table 2) (82).

DDX3, a protein of the DEAD-box RNA helicases family, is involved in miRNAs demethylation due to its interaction with AGO2 protein and is involved in miRNA synthesis and function (86). Furthermore, m6A-AGO2 transcripts influence cellular miRNA levels and induced cell senescence (87). The role of miRNA in spermatogenesis was strongly evidenced in the mouse testis (88). ALKBH5 deficiency caused aberrant mammalian spermatogenesis or apoptosis because of the removal of the m6A modification on mRNAs (9) (Figures 3B, C and Table 2).

\section{CIRCRNA AND N6-METHYLADENOSINE}

In recent research, circRNAs have ceased to be considered irrelevant artefacts of splicing errors, and are considered factors impacting the post-transcriptional regulation of gene expression These single-stranded RNAs can be detected in plasma, exosomes, and cell-free saliva (89). Mounting evidence has shown that m6A modification may participate in regulating the biological functions of circRNAs. circRNA may be a potential target for cancer therapy (Figure 4 and Table 3 ).

\section{1 m6A-Regulated miRNA/RBP Sponges of circRNA}

In addition to mRNAs, pseudogenic RNAs, or lncRNAs, circRNAs have also be defined as ceRNA and compete with miRNAs or RBPs (Figure 4A) (104). These competing transcripts probably crossregulate each other. In primary liver cancer, the highly expressed circRNA_104075 stimulated YAP expression both at the mRNA and protein levels (90). Further study has suggested that removal of the m6A modification on the $3^{\prime} \mathrm{UTR}$ region of YAP was essential for miR-582-3p binding to YAP. Furthermore, serum circ_104075 possessed high sensitivity and specificity for HCC diagnosis. circRNA-SORE sustains sorafenib resistance in HCC, and m6A modification increases the stability of circRNA-SORE (91). Yang et al. characterized eleven m6A containing circRNAs, among which seven circRNAs were significantly correlated with YTHDF2 (105). Furthermore, Chen et al. demonstrated that circFOREIGN can potentially activate gene expression of immunogenic factors (92). Nevertheless, YTHDF2 abrogated innate immunity by sequestering m6A-circRNA (Figure 4B and Table 3). The study established a patient-derived xenograft (PDX) tumor model for CRC and found that circNSUN2 strongly interfered with liver metastasis of CRC (93). The exon5-exon4 junction site of circNSUN2 was identified as the m6A modification site. The binding of YTHDC1 to the m6Amodified circNSUN2 promoted a complex nuclear to cytoplasmic export analogous to mRNA trafficking (Figure 4C and Table 3) (93). Interestingly, Wu et al. showed that METTL3 stabilized the expression of circCUX1 through the m6A methylation modification, which in turn, promoted radiotherapy resistance of hypopharyngeal cancer via caspase1 (94).

\section{2 m6A-Mediated Translation Potential of circRNA}

Some circRNAs carrying internal ribosome entry sites (IRESs) have the potential capacity of coding proteins (106). GranadosRiveron reported that a single m6A modification was sufficient to instigate the functional translation of circRNAs in the presence of eIF4G2 and YTHDF3 (107). Sun et al. found that circPVRL3 structure contained RRm6ACH ( $\mathrm{R}=\mathrm{G}$ or $\mathrm{A} ; \mathrm{H}=\mathrm{A}, \mathrm{C}$ or $\mathrm{U}), \mathrm{ORF}$, and IRES (95). Previous studies have documented that the m6A modification could regulate circRNA translation (108). Thus, it is reasonable to believe that circPVRL3 could translate into detectable peptides endogenously under certain cellular stresses. Zhao et al. reported that circE7 is found mainly in the cytoplasm and derives from oncogenic human papillomaviruses (HPVs) (96). CircE7 is highly m6A modified and translates to E7 oncoprotein following its association with polysomes.

\section{3 circRNAs-Mediated Regulation on m6A Effectors}

circRNAs also play a vital role in regulating the expression of m6Arelated proteins. It has been reported that circNDUFB2 is downregulated in NSCLC and inhibits the malignant progression of NSCLC (97). Mechanically, circNDUFB2 was found co-localized with IGF2BPs in the cytoplasm and physically interacted with IGF2BP1/2/3. Interestingly, overexpression of circNDUFB2 has no effect on the mRNA levels but protein levels of IGF2BPs. Further research found that circNDUFB2 reduces IGF2BPs stability via ubiquitin/proteasome-mediated degradation of IGF2BPs (97). circSTAG1 can bind with ALKBH5 in the astrocyte cytoplasm of the chronic unpredictable stress-treated mouse hippocampus, overexpressed circSTAG1 absorbs ALKBH5 and decreases the translocation of ALKBH5 into the nucleus, resulting in enhancement of m6A methylation on fatty acid amide hydrolase (FAAH) mRNA and subsequent degradation of FAAH (98). In addition, circMAPK4 acts as a ham-miR-139-5p sponge to regulate the expression and activity of YTHDF1 (99). circPTPRA can 


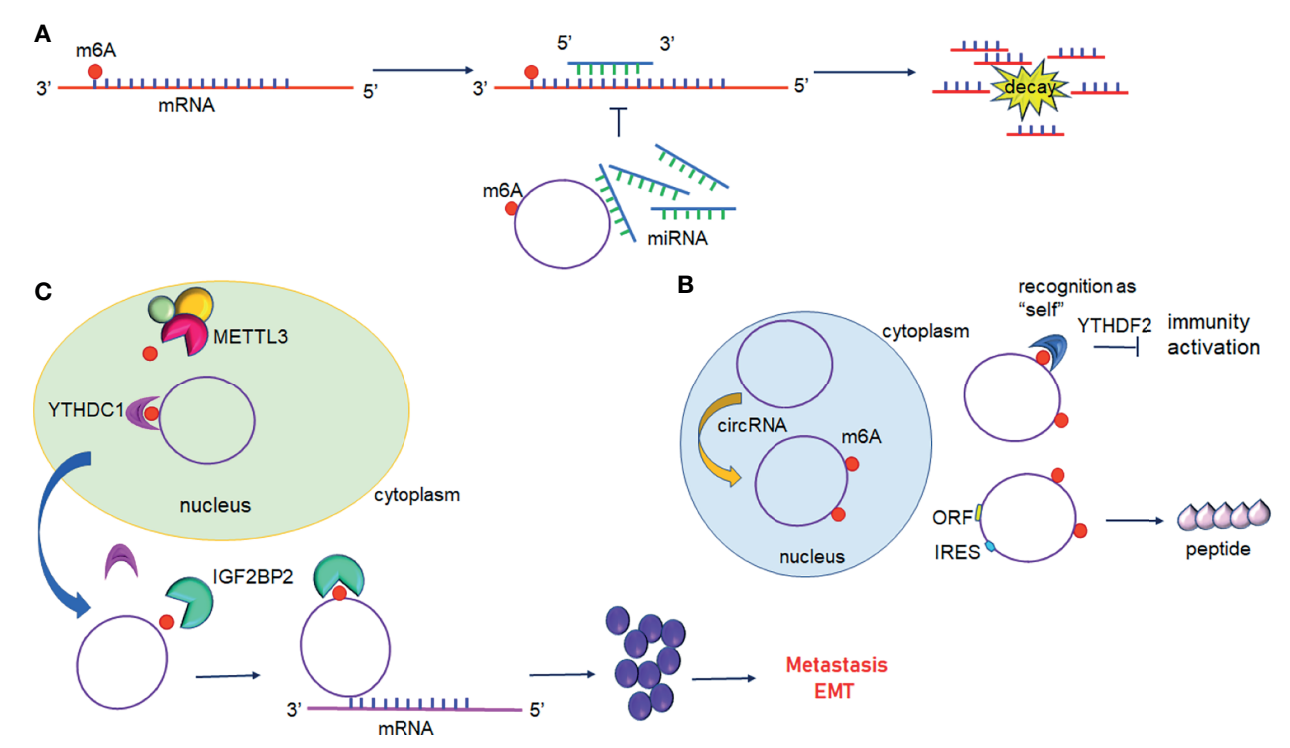

FIGURE 4 | Interplay between circular RNAs and m6A modification. (A) circRNAs act as ceRNA to regulate gene expression. m6A modified circRNAs sequester miRNAs resulting in inhibition of miRNA-direct gene silencing. (B) m6A modification of circRNAs suppresses immune genes expression or initiates translation. (C) m6A modified circRNAs regulate gene expression by directly targeting mRNA.YTHDC1 promotes the nuclear export of circRNAs to the cytoplasm following methylation by MEITL3. IGF2BP2 promotes the stability of m6A-circRNAs and interacts with oncogenic mRNAs.

directly bind to the $\mathrm{KH} 3$ and $\mathrm{KH} 4$ domains of IGF2BP1, and block recognition of m6A-modified MYC and FSCN1 transcripts in BC cells (100).

\section{OTHER NONCODING RNA AND M6A}

m6A modification may also occur on rRNA and spliceosome RNA, albeit the relevant mechanism is not as well-known as that of mRNA or the abovementioned ncRNA. Ma et al. reported that human $28 \mathrm{~S}$ rRNA undergoes m6A modification at position
A4220 by ZCCHC4, which is aberrantly expressed in tumor tissues and positively regulates HCC cancer cell proliferation (101). An additional rRNA m6A modification is located on A 1832 in 18s rRNA. These rRNAs m6A-modifications influence many aspects of ribosome activity, including localization, tertiary structure, and dynamics (109). In contrast to METTL3-METTL14-mediated m6A modification, METTL16 modified sites are primarily located in either introns or exonintron boundaries (49). METTL16 is responsible for the methylation of MAT2A mRNA and the spliceosome U6 snRNA $(49,102,103,110)$. U6 snRNA is crucial to pre-

TABLE 3 | CircRNAs in cancer or normal cells and related m6A effectors.

\begin{tabular}{|c|c|c|c|c|c|}
\hline Circ RNA & Cancer & Expression & Pathway & m6A effector & Reference \\
\hline Circ FOREIGN & Mammalian cells & - & $\begin{array}{l}\text { RIG-I } \\
\text { K63-Ub }\end{array}$ & $\begin{array}{l}\text { YTHDF2 } \\
\text { METTL3 }\end{array}$ & (92) \\
\hline Circ NSUN2 & $\mathrm{CRC}$ & Upregulation & HMGA2 & $\begin{array}{l}\text { YTHDC1 } \\
\text { IGF2BP2 }\end{array}$ & (93) \\
\hline Circ E7 & $\mathrm{CC}$ & - & HPVs & - & (96) \\
\hline circNDUFB2 & NSCLC & Downregulation & TRIM25 & IGF2BPs & (97) \\
\hline circSTAG1 & MDD & Downregulation & FAAH & ALKBH5 & (98) \\
\hline circMAPK4 & $\mathrm{HCC}$ & Upregulation & miR-139 & YTHDF1 & (99) \\
\hline circPTPRA & $\mathrm{BC}$ & Downregulation & MYC/FSCN1 & IGF2BP1 & (100) \\
\hline \multicolumn{6}{|l|}{ Others } \\
\hline
\end{tabular}

HPSCC, Hypopharyngeal squamous cell carcinoma; MDD, major depressive disorder. 
mRNAs splicing events. U6 snRNA carries m6A modification at position A43, an evolutionarily conserved nucleotide, and is lethal if mutated in yeast models (111).

\section{PERSPECTIVES}

In recent years, the emergence of transcriptomic approaches and bioinformatics have placed $\mathrm{m} 6 \mathrm{~A}$ modification to the research spotlight, and have expanded the full scope of m6A targets, including coding RNA and ncRNAs, and have convincingly elucidated their roles in multiple mammalian cell types. METTL16, a newfound m6A effector, acts as both a writer and U6 snRNA reader (112). In addition, METTL16 modified sites almost lie within introns, which is different from that of METTL3-METTL14 mediated sites, indicating a novel specific mechanism in methylation modifications. The dual identity of METTL16 deserves further exploration. During oxidative stress, NSUN2-mediated 5-methylcytosine (m5C) activity together with METTL3-METTL14-mediated m6A methylation contributes to synergistic upregulation of p21 (113). Methylation at m6A can facilitate the methylation at $\mathrm{m} 5 \mathrm{C}$, and vice versa. This functional interconnection between methylation sites leads to doubt about whether other chemical modifications can promote or inhibit coding RNA or ncRNAs at the posttranscriptional level.

In particular, m6A distribution mapping in lncRNAs is distinct compared to other mRNAs, but underlying effects remain currently unknown. Many lncRNAs are retained in the nucleus, which implies they exert their regulatory effects on the chromatin; however, this effect also can be impaired by m6A deposition. What are the effects of lncRNAs and m6A joint modulation of chromosomal conformations? Functionally, m6A-sculpted lncRNAs levels in the fetus are fewer than the mRNA counterparts, suggesting m6A-lncRNAs may be related to cell senescence in biological development. lncRNAs are expressed in a cell-type-dependent and tissue-specific-dependent manner. This pattern is conducive to the identification of novel landmarks for certain diseases, but also raises new questions about that how lncRNAs function in different environments, which warrants additional studies in the future. As Zuo et al. claimed, in xenograft tumors of HCC, the overall survival rate in mice was significantly prolonged following treatment with a PLGA-based siLINC00958 nanoplatform (34). The polymeric nanoparticle platform is formulated with poly (lactic acid/glycolic) copolymer (PLGA) and can deliver different drugs including siRNAs. This

\section{REFERENCES}

1. Roundtree IA, Evans ME, Pan T, He C. Dynamic RNA Modifications in Gene Expression Regulation. Cell (2017) 169:1187-200. doi: 10.1016/j.cell.2017.05.045

2. Meyer KD, Jaffrey SR. Rethinking M(6)A Readers, Writers, and Erasers. Annu Rev Cell Dev Biol (2017) 33:319-42. doi: 10.1146/annurev-cellbio-100616-060758

3. Qian JY, Gao J, Sun X, Cao MD, Shi L, Xia TS, et al. KIAA1429 Acts as an Oncogenic Factor in Breast Cancer by Regulating CDK1 in an N6Methyladenosine-Independent Manner. Oncogene (2019) 38:6123-41. doi: 10.1038/s41388-019-0861-z nanotherapy is unlike conventional therapies and exhibits limited systemic toxicity, low immunogenicity, good controllability, highefficiency, and tumor targeting. It is a promising approach to target disease mediated by the dysregulation of noncoding RNAs.

However, the study of m6A modification is still in its infancy, biological functions of the m6A-related protein family still need to be further explored. First, m6A modification can occur in most tumors, it is difficult to identify the specific m6A effectors in different tumors accurately. Second, there is no recognized reference method for the detection of m6A modification, methylated RNA immunoprecipitation combined with high throughput sequencing (MeRIP-Seq) can only identify the m6A hypermethylated region, but cannot quantitatively analyze m6A level or detect single-based m6A methylation. Third, the crosslink between m6A modification and noncoding RNAs is complicated, the specific binding sites between m6A methylation and ncRNA need further study. Potential biomarkers for diagnosis and adjuvant therapies will come from a better understanding of these action mechanisms.

\section{CONCLUSION}

The aberrant levels of m6A have been associated with multiple activities of ncRNAs, such as lncRNA metabolism, miRNA biogenesis, and circRNA translation, especially in eukaryotic organisms. Accumulating evidence has suggested that dysregulation of m6 A modification leads to multiple diseases including carcinomas. Therein, to a great extent, the complicated regulatory mechanisms involved remain uncharted territory. In this review, based on the current literature, we have described the crosstalk between m6A deposition and ncRNAs on determining cell development and fate. m6A-derived transcriptome topology broadens our horizons into the mechanisms underlying gene expression. Further studies are necessary to reveal the biochemical and structural basis of these ncRNAs and m6A modifications, to design selective inhibitors and potential therapies in the future.

\section{AUTHOR CONTRIBUTIONS}

All authors listed have made a substantial, direct, and intellectual contribution to the work and approved it for publication.

4. Patil DP, Chen CK, Pickering BF, Chow A, Jackson C, Guttman M, et al. M (6)A RNA Methylation Promotes XIST-Mediated Transcriptional Repression. Nature (2016) 537:369-73. doi: 10.1038/nature19342

5. Wen J, Lv R, Ma H, Shen H, He C, Wang J, et al. Zc3h13 Regulates Nuclear RNA M(6)A Methylation and Mouse Embryonic Stem Cell SelfRenewal. Mol Cell (2018) 69:1028-38.e6. doi: 10.1016/j.molcel. 2018.02.015

6. Pinello N, Sun S, Wong JJ. Aberrant Expression of Enzymes Regulating M (6)A mRNA Methylation: Implication in Cancer. Cancer Biol Med (2018) 15:323-34. doi: 10.20892/j.issn.2095-3941.2018.0365 
7. Warda AS, Kretschmer J, Hackert P, Lenz C, Urlaub H, Hobartner C, et al. Human METTL16 Is a N(6)-Methyladenosine M(6)A Methyltransferase That Targets Pre-mRNAs and Various Non-Coding RNAs. EMBO Rep (2017) 18:2004-14. doi: 10.15252/embr.201744940

8. Jia G, Fu Y, Zhao X, Dai Q, Zheng G, Yang Y, et al. N6-Methyladenosine in Nuclear RNA Is a Major Substrate of the Obesity-Associated FTO. Nat Chem Biol (2011) 7:885-7. doi: 10.1038/nchembio.687

9. Zheng G, Dahl JA, Niu Y, Fedorcsak P, Huang CM, Li CJ, et al. ALKBH5 Is a Mammalian RNA Demethylase That Impacts RNA Metabolism and Mouse Fertility. Mol Cell (2013) 49:18-29. doi: 10.1016/j.molcel.2012.10.015

10. Shi H, Wang X, Lu Z, Zhao BS, Ma H, Hsu PJ, et al. YTHDF3 Facilitates Translation and Decay of N(6)-Methyladenosine-Modified RNA. Cell Res (2017) 27:315-28. doi: 10.1038/cr.2017.15

11. Wojtas MN, Pandey RR, Mendel M, Homolka D, Sachidanandam R, Pillai RS. Regulation of M(6)A Transcripts by the 3' $\rightarrow$ ' RNA Helicase YTHDC2 Is Essential for a Successful Meiotic Program in the Mammalian Germline. Mol Cell (2017) 68:374-87.e12. doi: 10.1016/j.molcel.2017.09.021

12. Huang H, Weng H, Sun W, Qin X, Shi H, Wu H, et al. Recognition of RNA N(6)-Methyladenosine by IGF2BP Proteins Enhances mRNA Stability and Translation. Nat Cell Biol (2018) 20:285-95. doi: 10.1038/s41556-018-0045-Z

13. Edupuganti RR, Geiger S, Lindeboom RGH, Shi H, Hsu PJ, Lu Z, et al. N(6)Methyladenosine (M(6)A) Recruits and Repels Proteins to Regulate mRNA Homeostasis. Nat Struct Mol Biol (2017) 24:870-8. doi: 10.1038/nsmb.3462

14. Alarcon CR, Goodarzi H, Lee H, Liu X, Tavazoie S, Tavazoie SF. HNRNPA2B1 Is a Mediator of M(6)A-Dependent Nuclear RNA Processing Events. Cell (2015) 162:1299-308. doi: 10.1016/j.cell.2015.08.011

15. Patil DP, Pickering BF, Jaffrey SR. Reading M(6)A in the Transcriptome: $M$ (6)A-Binding Proteins. Trends Cell Biol (2018) 28:113-27. doi: 10.1016/ j.tcb.2017.10.001

16. Zhang J, Bai R, Li M, Ye H, Wu C, Wang C, et al. Excessive miR-25-3p Maturation via N(6)-Methyladenosine Stimulated by Cigarette Smoke Promotes Pancreatic Cancer Progression. Nat Commun (2019) 10:1858. doi: 10.1038/s41467-019-09712-x

17. Fazi F, Fatica A. Interplay Between N (6)-Methyladenosine (M(6)A) and Non-Coding RNAs in Cell Development and Cancer. Front Cell Dev Biol (2019) 7:116. doi: 10.3389/fcell.2019.00116

18. Park OH, Ha H, Lee $\mathrm{Y}$, Boo $\mathrm{SH}$, Kwon $\mathrm{DH}$, Song HK, et al. Endoribonucleolytic Cleavage of M(6)A-Containing RNAs by RNase P/ MRP Complex. Mol Cell (2019) 74:494-507.e8. doi: 10.1016/j.molcel. 2019.02.034

19. Wu Y, Yang X, Chen Z, Tian L, Jiang G, Chen F, et al. M(6)A-Induced IncRNA RP11 Triggers the Dissemination of Colorectal Cancer Cells via Upregulation of Zeb1. Mol Cancer (2019) 18:87. doi: 10.1186/s12943-0191014-2

20. Ji Z, Song R, Regev A, Struhl K. Many lncRNAs, 5'utrs, and Pseudogenes Are Translated and Some Are Likely to Express Functional Proteins. Elife (2015) 4:e08890. doi: 10.7554/eLife.08890

21. Xiao S, Cao S, Huang Q, Xia L, Deng M, Yang M, et al. The RNA N(6)Methyladenosine Modification Landscape of Human Fetal Tissues. Nat Cell Biol (2019) 21:651-61. doi: 10.1038/s41556-019-0315-4

22. Hammond SM. An Overview of microRNAs. Adv Drug Delivery Rev (2015) 87:3-14. doi: 10.1016/j.addr.2015.05.001

23. van der Kwast R, Quax PHA, Nossent AY. An Emerging Role for isomiRs and the microRNA Epitranscriptome in Neovascularization. Cells (2019) 9 (1):61. doi: $10.3390 /$ cells 9010061

24. Jie M, Feng T, Huang W, Zhang M, Feng Y, Jiang H, et al. Subcellular Localization of miRNAs and Implications in Cellular Homeostasis. Genes (Basel) (2021) 12(6):856. doi: 10.3390/genes 12060856

25. Hata A, Lieberman J. Dysregulation of microRNA Biogenesis and Gene Silencing in Cancer. Sci Signal (2015) 8:re3. doi: 10.1126/scisignal.2005825

26. Meng S, Zhou H, Feng Z, Xu Z, Tang Y, Li P, et al. CircRNA: Functions and Properties of a Novel Potential Biomarker for Cancer. Mol Cancer (2017) 16:94. doi: 10.1186/s12943-017-0663-2

27. Kristensen LS, Andersen MS, Stagsted LVW, Ebbesen KK, Hansen TB, Kjems J. The Biogenesis, Biology and Characterization of Circular RNAs. Nat Rev Genet (2019) 20:675-91. doi: 10.1038/s41576-019-0158-7

28. Ni W, Yao S, Zhou Y, Liu Y, Huang P, Zhou A, et al. Long Noncoding RNA GAS5 Inhibits Progression of Colorectal Cancer by Interacting With and
Triggering YAP Phosphorylation and Degradation and Is Negatively Regulated by the M(6)A Reader YTHDF3. Mol Cancer (2019) 18:143. doi: 10.1186/s12943-019-1079-y

29. Zhang J, Guo S, Piao HY, Wang Y, Wu Y, Meng XY, et al. ALKBH5 Promotes Invasion and Metastasis of Gastric Cancer by Decreasing Methylation of the lncRNA Neat1. J Physiol Biochem (2019) 75:379-89. doi: 10.1007/s13105-019-00690-8

30. Chen S, Zhou L, Wang Y. ALKBH5-Mediated M(6)A Demethylation of lncRNA PVT1 Plays an Oncogenic Role in Osteosarcoma. Cancer Cell Int (2020) 20:34. doi: 10.1186/s12935-020-1105-6

31. Hu X, Peng WX, Zhou H, Jiang J, Zhou X, Huang D, et al. IGF2BP2 Regulates DANCR by Serving as an N6-Methyladenosine Reader. Cell Death Differ (2020) 27:1782-94. doi: 10.1038/s41418-019-0461-Z

32. Zheng ZQ, Li ZX, Zhou GQ, Lin L, Zhang LL, Lv JW, et al. Long Noncoding RNA FAM225A Promotes Nasopharyngeal Carcinoma Tumorigenesis and Metastasis by Acting as ceRNA to Sponge miR-590-3p/miR-1275 and Upregulate ITGB3. Cancer Res (2019) 79:4612-26. doi: 10.1158/00085472.CAN-19-0799

33. Yang D, Qiao J, Wang G, Lan Y, Li G, Guo X, et al. N6-Methyladenosine Modification of lincRNA 1281 Is Critically Required for mESC Differentiation Potential. Nucleic Acids Res (2018) 46:3906-20. doi: 10.1093/nar/gky130

34. Zuo X, Chen Z, Gao W, Zhang Y, Wang J, Wang J, et al. M6A-Mediated Upregulation of LINC00958 Increases Lipogenesis and Acts as a Nanotherapeutic Target in Hepatocellular Carcinoma. J Hematol Oncol (2020) 13:5. doi: 10.1186/s13045-019-0839-x

35. Wu J, Pang R, Li M, Chen B, Huang J, Zhu Y. M6a-Induced IncRNA MEG3 Suppresses the Proliferation, Migration and Invasion of Hepatocellular Carcinoma Cell Through miR-544b/BTG2 Signaling. Onco Targets Ther (2021) 14:3745-55. doi: 10.2147/OTT.S289198

36. Lang C, Yin C, Lin K, Li Y, Yang Q, Wu Z, et al. M(6) A Modification of lncRNA PCAT6 Promotes Bone Metastasis in Prostate Cancer Through IGF2BP2-Mediated IGF1R mRNA Stabilization. Clin Transl Med (2021) 11: e426. doi: $10.1002 / \mathrm{ctm} 2.426$

37. Yuan X, Shi L, Guo Y, Sun J, Miao J, Shi J, et al. METTL3 Regulates Ossification of the Posterior Longitudinal Ligament via the lncRNA XIST/ miR-302a-3p/USP8 Axis. Front Cell Dev Biol (2021) 9:629895. doi: 10.3389/ fcell.2021.629895

38. Yan J, Huang X, Zhang X, Chen Z, Ye C, Xiang W, et al. LncRNA LINC00470 Promotes the Degradation of PTEN mRNA to Facilitate Malignant Behavior in Gastric Cancer Cells. Biochem Biophys Res Commun (2020) 521:887-93. doi: 10.1016/j.bbrc.2019.11.016

39. Hu H, Kong Q, Huang XX, Zhang HR, Hu KF, Jing Y, et al. Longnon-Coding RNA BLACAT2 Promotes Gastric Cancer Progression via the miR-193b-5p/ METTL3 Pathway. J Cancer (2021) 12:3209-21. doi: 10.7150/jca.50403

40. Shang W, Gao Y, Tang Z, Zhang Y, Yang R. The Pseudogene Olfr29-Ps1 Promotes the Suppressive Function and Differentiation of Monocytic MDSCs. Cancer Immunol Res (2019) 7:813-27. doi: 10.1158/23266066.CIR-18-0443

41. Rong D, Dong Q, Qu H, Deng X, Gao F, Li Q, et al. M(6)A-Induced LINC00958 Promotes Breast Cancer Tumorigenesis via the miR-378a-3p/ YY1 Axis. Cell Death Discov (2021) 7:27. doi: 10.1038/s41420-020-00382-Z

42. Wang X, Zhang J, Wang Y. Long Noncoding RNA GAS5-AS1 Suppresses Growth and Metastasis of Cervical Cancer by Increasing GAS5 Stability. Am J Transl Res (2019) 11:4909-21.

43. Zhang S, Zhao BS, Zhou A, Lin K, Zheng S, Lu Z, et al. M(6)A Demethylase ALKBH5 Maintains Tumorigenicity of Glioblastoma Stem-Like Cells by Sustaining FOXM1 Expression and Cell Proliferation Program. Cancer Cell (2017) 31:591-606.e6. doi: 10.1016/j.ccell.2017.02.013

44. He Y, Hu H, Wang Y, Yuan H, Lu Z, Wu P, et al. ALKBH5 Inhibits Pancreatic Cancer Motility by Decreasing Long Non-Coding RNA KCNK15-AS1 Methylation. Cell Physiol Biochem (2018) 48:838-46. doi: $10.1159 / 000491915$

45. Xue L, Li J, Lin Y, Liu D, Yang Q, Jian J, et al. M(6) A Transferase METTL3Induced lncRNA ABHD11-AS1 Promotes the Warburg Effect of Non-SmallCell Lung Cancer. J Cell Physiol (2021) 236:2649-58. doi: 10.1002/jcp.30023

46. Zhu L, Zhu Y, Han S, Chen M, Song P, Dai D, et al. Impaired Autophagic Degradation of lncRNA ARHGAP5-AS1 Promotes Chemoresistance in 
Gastric Cancer. Cell Death Dis (2019) 10:383. doi: 10.1038/s41419-019$1585-2$

47. Li N, Wang Y, Liu X, Luo P, Jing W, Zhu M, et al. Identification of Circulating Long Noncoding RNA HOTAIR as a Novel Biomarker for Diagnosis and Monitoring of Non-Small Cell Lung Cancer. Technol Cancer Res Treat (2017) 16:1060-6. doi: 10.1177/1533034617723754

48. Brown JA, Kinzig CG, DeGregorio SJ, Steitz JA. Methyltransferase-Like Protein 16 Binds the 3'-Terminal Triple Helix of MALAT1 Long Noncoding RNA. Proc Natl Acad Sci U S A (2016) 113:14013-8. doi: 10.1073/ pnas. 1614759113

49. Satterwhite ER, Mansfield KD. RNA Methyltransferase METTL16: Targets and Function. Wiley Interdiscip Rev RNA (2021) 10(1):e1681. doi: 10.1002/ wrna.1681

50. Liu N, Zhou KI, Parisien M, Dai Q, Diatchenko L, Pan T. N6Methyladenosine Alters RNA Structure to Regulate Binding of a LowComplexity Protein. Nucleic Acids Res (2017) 45:6051-63. doi: 10.1093/nar/ gkx141

51. Cao J, Mu Q, Huang H. The Roles of Insulin-Like Growth Factor 2 mRNABinding Protein 2 in Cancer and Cancer Stem Cells. Stem Cells Int (2018) 2018:4217259. doi: 10.1155/2018/4217259

52. Guo E, Liang C, He X, Song G, Liu H, Lv Z, et al. Long Noncoding RNA LINC00958 Accelerates Gliomagenesis Through Regulating miR-203/Cdk2. DNA Cell Biol (2018) 37:465-72. doi: 10.1089/dna.2018.4163

53. Wong CM, Tsang FH, Ng IO. Non-Coding RNAs in Hepatocellular Carcinoma: Molecular Functions and Pathological Implications. Nat Rev Gastroenterol Hepatol (2018) 15:137-51. doi: 10.1038/nrgastro.2017.169

54. Chokkalla AK, Mehta SL, Kim T, Chelluboina B, Kim J, Vemuganti R. Transient Focal Ischemia Significantly Alters the M(6)A Epitranscriptomic Tagging of RNAs in the Brain. Stroke (2019) 50:2912-21. doi: 10.1161/ STROKEAHA.119.026433

55. Yang C, Fan Z, Yang J. M(6)A Modification of LncRNA MALAT1: A Novel Therapeutic Target for Myocardial Ischemia-Reperfusion Injury. Int $J$ Cardiol (2020) 306:162. doi: 10.1016/j.ijcard.2019.11.140

56. Wang Y, Jing W, Ma W, Liang C, Chai H, Tu J. Down-Regulation of Long Non-Coding RNA GAS5-AS1 and Its Prognostic and Diagnostic Significance in Hepatocellular Carcinoma. Cancer biomark (2018) 22:22736. doi: 10.3233/CBM-170781

57. Wu Y, Lyu H, Liu H, Shi X, Song Y, Liu B. Downregulation of the Long Noncoding RNA GAS5-AS1 Contributes to Tumor Metastasis in Non-Small Cell Lung Cancer. Sci Rep (2016) 6:31093. doi: 10.1038/srep31093

58. Dinescu S, Ignat S, Lazar AD, Constantin C, Neagu M, Costache M. Epitranscriptomic Signatures in lncRNAs and Their Possible Roles in Cancer. Genes (Basel) (2019) 10(1):52. doi: 10.3390/genes10010052

59. Alarcon CR, Lee H, Goodarzi H, Halberg N, Tavazoie SF. N6Methyladenosine Marks Primary microRNAs for Processing. Nature (2015) 519:482-5. doi: 10.1038/nature14281

60. Han J, Wang JZ, Yang X, Yu H, Zhou R, Lu HC, et al. METTL3 Promote Tumor Proliferation of Bladder Cancer by Accelerating Pri-Mir221/222 Maturation in M6a-Dependent Manner. Mol Cancer (2019) 18:110. doi: 10.1186/s12943-019-1036-9

61. Garofalo M, Quintavalle C, Romano G, Croce CM, Condorelli G. Mir221/ 222 in Cancer: Their Role in Tumor Progression and Response to Therapy. Curr Mol Med (2012) 12:27-33. doi: 10.2174/156652412798376170

62. Chen Y, Zaman MS, Deng G, Majid S, Saini S, Liu J, et al. MicroRNAs 221/ 222 and Genistein-Mediated Regulation of ARHI Tumor Suppressor Gene in Prostate Cancer. Cancer Prev Res (Phila) (2011) 4:76-86. doi: 10.1158/ 1940-6207.CAPR-10-0167

63. Mardente S, Mari E, Massimi I, Fico F, Faggioni A, Pulcinelli F, et al. HMGB1-Induced Cross Talk Between PTEN and miRs 221/222 in Thyroid Cancer. BioMed Res Int (2015) 2015:512027. doi: 10.1155/2015/512027

64. Peng W, Li J, Chen R, Gu Q, Yang P, Qian W, et al. Upregulated METTL3 Promotes Metastasis of Colorectal Cancer via miR-1246/SPRED2/MAPK Signaling Pathway. J Exp Clin Cancer Res (2019) 38:393. doi: 10.1186/ s13046-019-1408-4

65. Huang S, Luo S, Gong C, Liang L, Xiao Y, Li M, et al. MTTL3 Upregulates microRNA-1246 to Promote Occurrence and Progression of NSCLC via Targeting Paternally Expressed Gene 3. Mol Ther Nucleic Acids (2021) 24:542-53. doi: 10.1016/j.omtn.2021.02.020
66. Liu E, Lv L, Zhan Y, Ma Y, Feng J, He Y, et al. METTL3/N6Methyladenosine/ miR-21-5p Promotes Obstructive Renal Fibrosis by Regulating Inflammation Through SPRY1/ERK/NF-kappaB Pathway Activation. J Cell Mol Med (2021) 25(16):7660-74. doi: 10.1111/jcmm.16603

67. Pan X, Hong X, Li S, Meng P, Xiao F. METTL3 Promotes Adriamycin Resistance in MCF-7 Breast Cancer Cells by Accelerating pri-microRNA221-3p Maturation in a M6a-Dependent Manner. Exp Mol Med (2021) 53:91-102. doi: 10.1038/s12276-020-00510-w

68. Wang H, Deng Q, Lv Z, Ling Y, Hou X, Chen Z, et al. N6-Methyladenosine Induced miR-143-3p Promotes the Brain Metastasis of Lung Cancer via Regulation of VASH1. Mol Cancer (2019) 18:181. doi: 10.1186/s12943-019$1108-\mathrm{x}$

69. Wang J, Ishfaq M, Xu L, Xia C, Chen C, Li J. METTL3/m(6)A/miRNA-873$5 p$ Attenuated Oxidative Stress and Apoptosis in Colistin-Induced Kidney Injury by Modulating Keap1/Nrf2 Pathway. Front Pharmacol (2019) 10:517. doi: 10.3389/fphar.2019.00517

70. Yang Y, Song S, Meng Q, Wang L, Li X, Xie S, et al. Mir24-2 Accelerates Progression of Liver Cancer Cells by Activating Pim1 Through TriMethylation of Histone H3 on the Ninth Lysine. J Cell Mol Med (2020) 24:2772-90. doi: 10.1111/jcmm.15030

71. Ma JZ, Yang F, Zhou CC, Liu F, Yuan JH, Wang F, et al. METTL14 Suppresses the Metastatic Potential of Hepatocellular Carcinoma by Modulating N(6) -Methyladenosine-Dependent Primary MicroRNA Processing. Hepatology (2017) 65:529-43. doi: 10.1002/hep.28885

72. Chen X, Xu M, Xu X, Zeng K, Liu X, Sun L, et al. METTL14 Suppresses CRC Progression via Regulating N6-Methyladenosine-Dependent Primary miR-375 Processing. Mol Ther (2020) 28:599-612. doi: 10.1016/j.ymthe.2019.11.016

73. Yang Z, Li J, Feng G, Gao S, Wang Y, Zhang S, et al. MicroRNA-145 Modulates N(6)-Methyladenosine Levels by Targeting the 3'-Untranslated mRNA Region of the N(6)-Methyladenosine Binding YTH Domain Family 2 Protein. J Biol Chem (2017) 292:3614-23. doi: 10.1074/jbc.M116.749689

74. Liu T, Zhou H, Lu H, Luo C, Wang Q, Peng Y, et al. MiR-4729 Regulates TIE1 mRNA M6a Modification and Angiogenesis in Hemorrhoids by Targeting METTL14. Ann Transl Med (2021) 9:232. doi: 10.21037/atm20-3399

75. Sun Z, Wang H, Wang Y, Yuan G, Yu X, Jiang H, et al. MiR-103-3p Targets the M(6) A Methyltransferase METTL14 to Inhibit Osteoblastic Bone Formation. Aging Cell (2021) 20:e13298. doi: 10.1111/acel.13298

76. Cui X, Wang Z, Li J, Zhu J, Ren Z, Zhang D, et al. Cross Talk Between RNA N6-Methyladenosine Methyltransferase-Like 3 and miR-186 Regulates Hepatoblastoma Progression Through Wnt/beta-Catenin Signalling Pathway. Cell Prolif (2020) 53:e12768. doi: 10.1111/cpr.12768

77. Zhang F, Yan Y, Cao X, Zhang J, Li Y, Guo C. Methylation of microRNA338-5p by EED Promotes METTL3-Mediated Translation of Oncogene CDCP1 in Gastric Cancer. Aging (Albany NY) (2021) 13:12224-38. doi: 10.18632/aging.103822

78. Song Z, Jia G, Ma P, Cang S. Exosomal miR-4443 Promotes Cisplatin Resistance in Non-Small Cell Lung Carcinoma by Regulating FSP1 M6a Modification-Mediated Ferroptosis. Life Sci (2021) 276:119399. doi: 10.1016/ j.lfs.2021.119399

79. Du M, Zhang Y, Mao Y, Mou J, Zhao J, Xue Q, et al. MiR-33a Suppresses Proliferation of NSCLC Cells via Targeting METTL3 mRNA. Biochem Biophys Res Commun (2017) 482:582-9. doi: 10.1016/j.bbrc.2016.11.077

80. Wei W, Huo B, Shi X. miR-600 Inhibits Lung Cancer via Downregulating the Expression of METTL3. Cancer Manag Res (2019) 11:1177-87. doi: 10.2147/CMAR.S181058

81. Xi Z, Wang P, Xue Y, Shang C, Liu X, Ma J, et al. Overexpression of miR-29a Reduces the Oncogenic Properties of Glioblastoma Stem Cells by Downregulating Quaking Gene Isoform 6. Oncotarget (2017) 8:24949-63. doi: $10.18632 /$ oncotarget.15327

82. Cai X, Wang X, Cao C, Gao Y, Zhang S, Yang Z, et al. HBXIP-Elevated Methyltransferase METTL3 Promotes the Progression of Breast Cancer via Inhibiting Tumor Suppressor Let-7g. Cancer Lett (2018) 415:11-9. doi: 10.1016/j.canlet.2017.11.018

83. Klinge CM, Piell KM, Tooley CS, Rouchka EC. HNRNPA2/B1 Is Upregulated in Endocrine-Resistant LCC9 Breast Cancer Cells and Alters the miRNA Transcriptome When Overexpressed in MCF-7 Cells. Sci Rep (2019) 9:9430. doi: 10.1038/s41598-019-45636-8 
84. Gualdrini F, Esnault C, Horswell S, Stewart A, Matthews N, Treisman R. SRF Co-Factors Control the Balance Between Cell Proliferation and Contractility. Mol Cell (2016) 64:1048-61. doi: 10.1016/j.molcel.2016.10.016

85. Muller S, Glass M, Singh AK, Haase J, Bley N, Fuchs T, et al. IGF2BP1 Promotes SRF-Dependent Transcription in Cancer in a M6a- and miRNA-Dependent Manner. Nucleic Acids Res (2019) 47:375-90. doi: 10.1093/nar/gky1012

86. Shah A, Rashid F, Awan HM, Hu S, Wang X, Chen L, et al. The DEAD-Box RNA Helicase DDX3 Interacts With M(6)A RNA Demethylase ALKBH5. Stem Cells Int (2017) 2017:8596135. doi: 10.1155/2017/8596135

87. Min KW, Zealy RW, Davila S, Fomin M, Cummings JC, Makowsky D, et al. Profiling of M6a RNA Modifications Identified an Age-Associated Regulation of AGO2 mRNA Stability. Aging Cell (2018) 17:e12753. doi: 10.1111/acel.12753

88. Chen X, Li X, Guo J, Zhang P, Zeng W. The Roles of microRNAs in Regulation of Mammalian Spermatogenesis. J Anim Sci Biotechnol (2017) 8:35. doi: 10.1186/s40104-017-0166-4

89. Yang Y, Fan X, Mao M, Song X, Wu P, Zhang Y, et al. Extensive Translation of Circular RNAs Driven by N(6)-Methyladenosine. Cell Res (2017) 27:62641. doi: 10.1038/cr.2017.31

90. Zhang X, Xu Y, Qian Z, Zheng W, Wu Q, Chen Y, et al. circRNA_104075 Stimulates YAP-Dependent Tumorigenesis Through the Regulation of HNF4a and may Serve as a Diagnostic Marker in Hepatocellular Carcinoma. Cell Death Dis (2018) 9:1091. doi: 10.1038/s41419-018-1132-6

91. Xu J, Wan Z, Tang M, Lin Z, Jiang S, Ji L, et al. N(6)-MethyladenosineModified circRNA-SORE Sustains Sorafenib Resistance in Hepatocellular Carcinoma by Regulating Beta-Catenin Signaling. Mol Cancer (2020) 19:163. doi: 10.1186/s12943-020-01281-8

92. Chen YG, Chen R, Ahmad S, Verma R, Kasturi SP, Amaya L, et al. N6Methyladenosine Modification Controls Circular RNA Immunity. Mol Cell (2019) 76:96-109.e9. doi: 10.1016/j.molcel.2019.07.016

93. He RZ, Jiang J, Luo DX. M6A Modification of Circnsun2 Promotes Colorectal Liver Metastasis. Genes Dis (2021) 8:6-7. doi: 10.1016/j.gendis 2019.12.002

94. Wu P, Fang X, Liu Y, Tang Y, Wang W, Li X, et al. N6-Methyladenosine Modification of Circcuxl Confers Radioresistance of Hypopharyngeal Squamous Cell Carcinoma Through Caspase1 Pathway. Cell Death Dis (2021) 12:298. doi: 10.1038/s41419-021-03558-2

95. Sun HD, Xu ZP, Sun ZQ, Zhu B, Wang Q, Zhou J, et al. Down-Regulation of Circpvrl3 Promotes the Proliferation and Migration of Gastric Cancer Cells. Sci Rep (2018) 8:10111. doi: 10.1038/s41598-018-27837-9

96. Zhao J, Lee EE, Kim J, Yang R, Chamseddin B, Ni C, et al. Transforming Activity of an Oncoprotein-Encoding Circular RNA From Human Papillomavirus. Nat Commun (2019) 10:2300. doi: 10.1038/s41467-019-10246-5

97. Li B, Zhu L, Lu C, Wang C, Wang H, Jin H, et al. Circndufb2 Inhibits NonSmall Cell Lung Cancer Progression via Destabilizing IGF2BPs and Activating Anti-Tumor Immunity. Nat Commun (2021) 12:295. doi: 10.1038/s41467-020-20527-z

98. Huang R, Zhang Y, Bai Y, Han B, Ju M, Chen B, et al. N(6)-Methyladenosine Modification of Fatty Acid Amide Hydrolase Messenger RNA in Circular RNA STAG1-Regulated Astrocyte Dysfunction and Depressive-Like Behaviors. Biol Psychiatry (2020) 88:392-404. doi: 10.1016/j.biopsych.2020.02.018

99. Chi F, Cao Y, Chen Y. Analysis and Validation of circRNA-miRNA Network in Regulating M(6)A RNA Methylation Modulators Reveals Circmap2k4/ miR-139-5p/YTHDF1 Axis Involving the Proliferation of Hepatocellular Carcinoma. Front Oncol (2021) 11:560506. doi: 10.3389/fonc.2021.560506

100. Xie F, Huang C, Liu F, Zhang H, Xiao X, Sun J, et al. CircPTPRA Blocks the Recognition of RNA N(6)-Methyladenosine Through Interacting With IGF2BP1 to Suppress Bladder Cancer Progression. Mol Cancer (2021) 20:68. doi: 10.1186/s12943-021-01359-x
101. Ma H, Wang X, Cai J, Dai Q, Natchiar SK, Lv R, et al. N(6)-Methyladenosine Methyltransferase ZCCHC4 Mediates Ribosomal RNA Methylation. Nat Chem Biol (2019) 15:88-94. doi: 10.1038/s41589-018-0184-3

102. Pendleton KE, Chen B, Liu K, Hunter OV, Xie Y, Tu BP, et al. The U6 snRNA M(6)A Methyltransferase METTL16 Regulates SAM Synthetase Intron Retention. Cell (2017) 169:824-35.e14. doi: 10.1016/j.cell.2017.05.003

103. Aoyama T, Yamashita S, Tomita K. Mechanistic Insights Into M6a Modification of U6 snRNA by Human METTL16. Nucleic Acids Res (2020) 48:5157-68. doi: 10.1093/nar/gkaa227

104. Ashwal-Fluss R, Meyer M, Pamudurti NR, Ivanov A, Bartok O, Hanan M, et al. circRNA Biogenesis Competes With pre-mRNA Splicing. Mol Cell (2014) 56:55-66. doi: 10.1016/j.molcel.2014.08.019

105. Yang J, Han Q, Li C, Yang H, Chen X, Wang X. Circular RNA Circ 0001105 Inhibits Progression and Metastasis of Osteosarcoma by Sponging miR-766 and Activating YTHDF2 Expression. Onco Targets Ther (2020) 13:1723-36. doi: 10.2147/OTT.S234668

106. Wang Y, Wang Z. Efficient Backsplicing Produces Translatable Circular mRNAs. RNA (2015) 21:172-9. doi: 10.1261/rna.048272.114

107. Granados-Riveron JT, Aquino-Jarquin G. The Complexity of the Translation Ability of circRNAs. Biochim Biophys Acta (2016) 1859:1245-51. doi: 10.1016/j.bbagrm.2016.07.009

108. Zhou C, Molinie B, Daneshvar K, Pondick JV, Wang J, Van Wittenberghe N, et al. Genome-Wide Maps of M6a circRNAs Identify Widespread and CellType-Specific Methylation Patterns That are Distinct From mRNAs. Cell Rep (2017) 20:2262-76. doi: 10.1016/j.celrep.2017.08.027

109. Dang W, Xie Y, Cao P, Xin S, Wang J, Li S, et al. N(6)-Methyladenosine and Viral Infection. Front Microbiol (2019) 10:417. doi: 10.3389/fmicb. 2019.00417

110. Watabe E, Togo-Ohno M, Ishigami Y, Wani S, Hirota K, Kimura-Asami M, et al. M(6) A-Mediated Alternative Splicing Coupled With NonsenseMediated mRNA Decay Regulates SAM Synthetase Homeostasis. EMBO J (2021) 40(14):e106434. doi: 10.15252/embj.2020106434

111. Ruszkowska A, Ruszkowski M, Dauter Z, Brown JA. Structural Insights Into the RNA Methyltransferase Domain of METTL16. Sci Rep (2018) 8:5311. doi: $10.1038 / \mathrm{s} 41598-018-23608-8$

112. Nance DJ, Satterwhite ER, Bhaskar B, Misra S, Carraway KR, Mansfield KD. Characterization of METTL16 as a Cytoplasmic RNA Binding Protein. PLoS One (2020) 15:e0227647. doi: 10.1371/journal.pone.0227647

113. Li Q, Li X, Tang H, Jiang B, Dou Y, Gorospe M, et al. NSUN2-Mediated M5c Methylation and METTL3/METTL14-Mediated M6a Methylation Cooperatively Enhance P21 Translation. J Cell Biochem (2017) 118:258798. doi: $10.1002 / j \mathrm{jcb} .25957$

Conflict of Interest: The authors declare that the research was conducted in the absence of any commercial or financial relationships that could be construed as a potential conflict of interest.

Publisher's Note: All claims expressed in this article are solely those of the authors and do not necessarily represent those of their affiliated organizations, or those of the publisher, the editors and the reviewers. Any product that may be evaluated in this article, or claim that may be made by its manufacturer, is not guaranteed or endorsed by the publisher.

Copyright (c) 2021 Zhang, Wei, Wang and Dong. This is an open-access article distributed under the terms of the Creative Commons Attribution License (CC BY). The use, distribution or reproduction in other forums is permitted, provided the original author(s) and the copyright owner(s) are credited and that the original publication in this journal is cited, in accordance with accepted academic practice. No use, distribution or reproduction is permitted which does not comply with these terms. 